\title{
Rapid Mapping for German Federal Authorities
}

\author{
Karsten Schmidt $^{\mathrm{a}}{ }^{*}$, Marian Graumann $^{\mathrm{b}}$, Joachim Bobrich $^{\mathrm{c}}$ \\ ${ }^{a}$ Federal Agency for Cartography and Geodesy, karsten.schmidt@bkg.bund.de \\ ${ }^{b}$ Federal Agency for Cartography and Geodesy, marian.graumann@bkg.bund.de \\ ${ }^{c}$ Federal Agency for Cartography and Geodesy, joachim.bobrich@bkg.bund.de \\ * Corresponding author
}

Keywords: thematic maps, rapid mapping, crisis management, GIS, map production

Abstract:

The Federal Agency for Cartography and Geodesy (BKG) in Germany is a technical agency under the Federal Ministry of the Interior, Building and Community (BMI). The organization consists of three departments namely Geoinformation, Geodesy and a Business Operations/Support department. Each department has multiple units which carry out their own respective duty in their field of subject. Tasks related to providing and homogenizing spatial data are located inside the geoinformation department. The main focus lies here on uniting the data collected by the federal states to form different types of nationwide datasets. The geodesy department focusses on real time satellite navigation systems, very long baseline interferometry and geodetic reference systems.

A recent development in the federal authorities has been the demand for quick maps and spatial data to support fast decision making processes. The BKG has established a complete workflow which allows for rapid data processing and the distribution to the respective customers. It is split into six sub processes with several branching options, processing and backpropagation steps. This allows for a systematic approach to covering the customer's demand for spatial data and for the internal production chain. The workflow is backed up by a project management system, a quality management system and a production management system each covered in a handbook specially prepared to ensure consistency along the workflow.

The workflow gets started by the customer posing their request either via the service center (SC) unit, via mail or phone or through the controlling institution as a decree or an executive order. The first contact is crucial as important information is being exchanged and a rough concept of the demand is shaped. Once the actual task has reached the BKG a ticket number and an internal processing identifier will be assigned by the SC and the resource manager (RM) respectively. These unique keys will be used for the communication with the customer and additionally utilized as a tool for the administrative actions being carried out in the background. This action involves querying the license status of the customer which in addition determines the maximum cost of the task and the authorization of the data being used. In parallel the task, now referred to as a ticket, is being implemented in the project management system. Certain additional information will be assigned to the ticket like the project manager (PM) and the project processor. Once this step has been completed the working directory for the ticket will be created by using a predefined folder structure. This structure is composed of a data, export, communications and documentation folder. Depending on the nature of the ticket various preconfigured templates are at hand and will be used to ensure uniformity of the final products. If needed further communication with the customer is carried out. Data research is the last step in this part of the workflow (see figure 1).

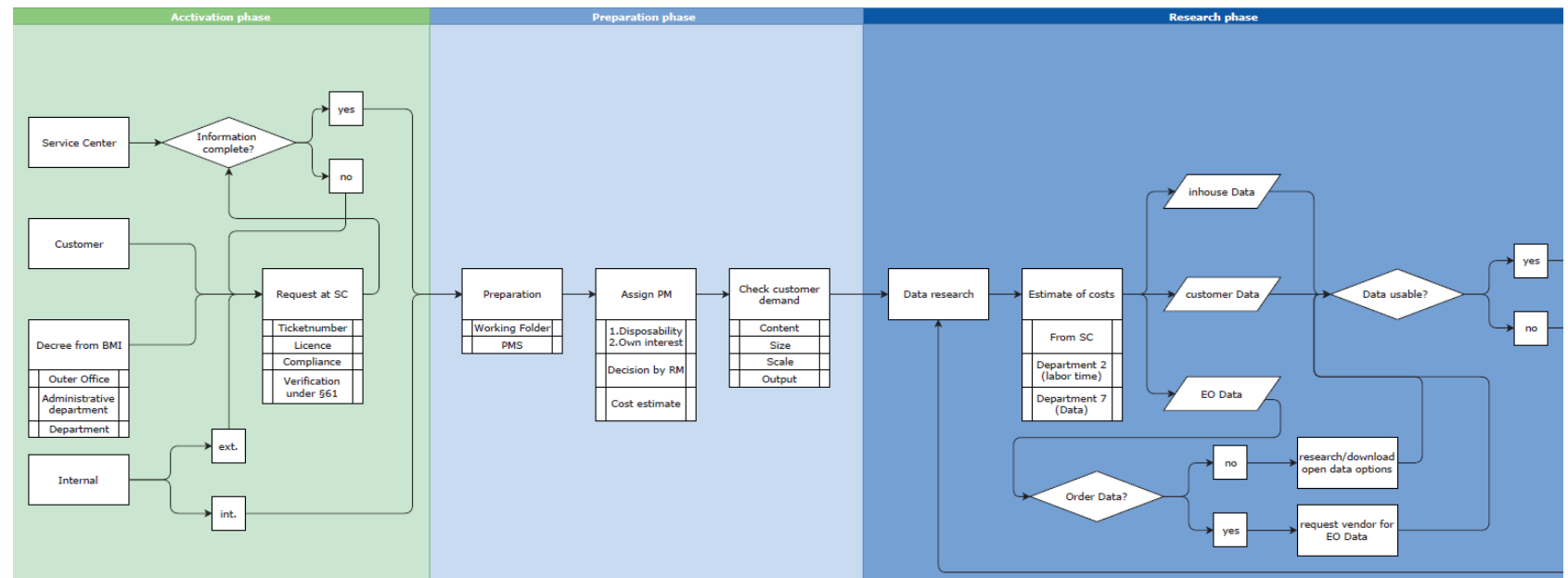

Figure 1. Extract from the workflow scheme: Activation Phase, Preparation Phase, Research Phase 
The processing of the actual data makes up most of the workflow regarding time. Aspects like a uniform styleguide and quality controls by colleagues are performed alongside the work progress. If any non comforming aspect is found, the feedback is given directly to the person in charge of the ticket while also being mirrored in the project management system. Once the product is ready for deployment, final communication with the customer is executed. Additional changes stated by the customer can be implemented whereby an extra iteration of the quality process will be necessary. The data deployment is either carried out as a print product, a DVD, an OGC service or via a download option (see figure 2).

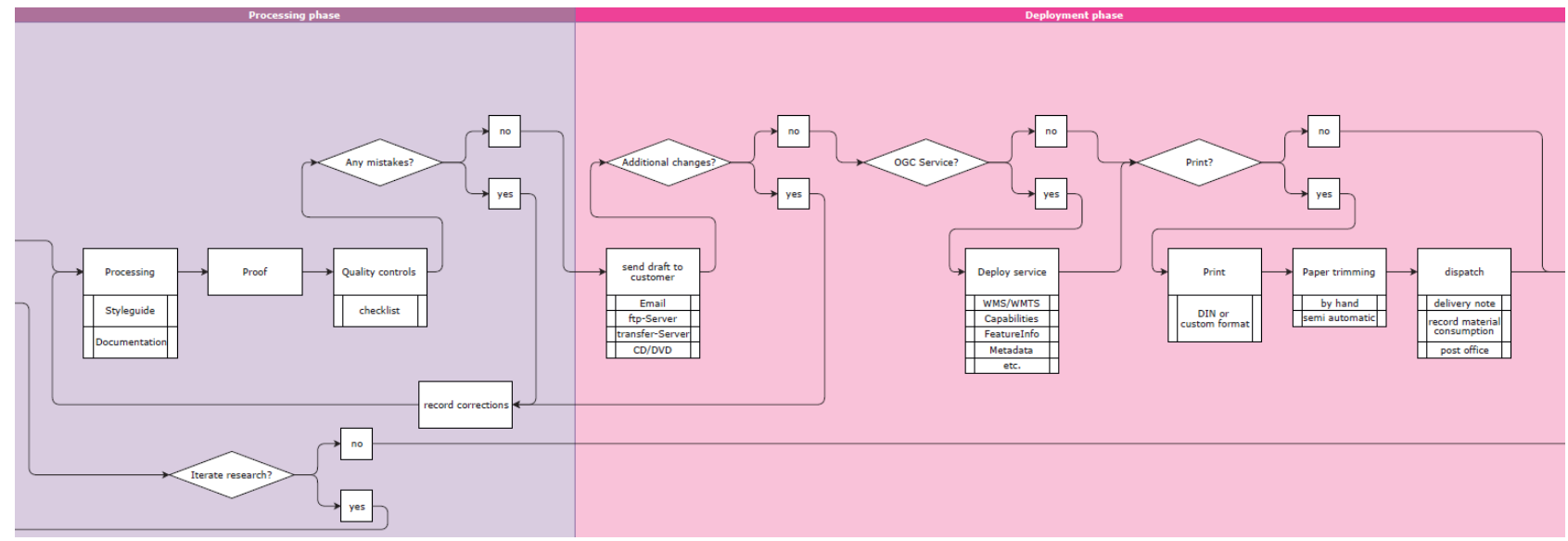

Figure 2. Extract from the workflow scheme: Processing Phase, Deployment Phase

The final steps of the workflow involve the report of delivery to the service center, the creation of the cost report and the closure of the ticket in the project management system. The cost report is created in a semi-automatic process from data stored in the project management system and combined with the material cost report. It is then send via e-mail to the service center which will carry out any necessary invoice collections. Furthermore the internal data folder structure is cleaned up and a screenshot is taken of the product to use it as a subsidiary product in the dedicated online gallery of created products. Finally the closing information is relayed to the service center and the ticket gets closed (see figure 3 ). This whole workflow can be executed within several hours, days or weeks, depending on the deadline imposed by the customer or the event. Actual rapid mapping activations are done by multiple people simultaneously working on different steps of the previously explained workflow. This allows for fast response times and the accomplishment of the activation in time.

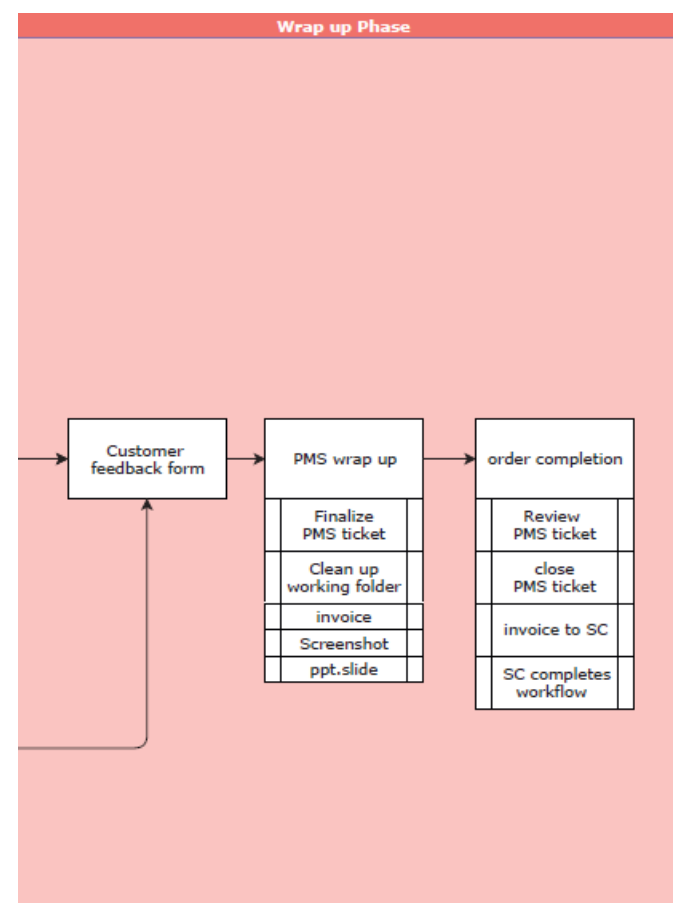

Figure 3. Extract from the workflow scheme: Wrap Up Phase 